\title{
Die Teilprivatisierung der Berliner Wasserbetriebe: Erfolgsmodell oder Abwicklungsfall?
}

Daseinsvorsorge; Öffentliche Ver- und Entsorger; Public Corporate Governance; Teilprivatisierung; Kommunale Wasserwirtschaft; Demokratische Steuerung

Die Teilprivatisierung der Berliner Wasserbetriebe (BWB) 1999 galt lange Zeit als Paradebeispiel für eine gelungene Public-Private-Partnership-Lösung. Dabei wurde vor allem hervorgehoben, dass diese rechtsformwahrende Privatisierung die Potenziale der öffentlich-rechtlichen Unternehmensform Anstalt mit den Effizienzkriterien des Privatsektors vorbildlich verbunden habe. Zwölf Jahre nach der Privatisierung wird in diesem Artikel eine Zwischenbilanz versucht.

\section{Hintergrund der Teilprivatisierung}

\section{Kommunale Finanznot, Entstaatlichungseuphorie, neoliberaler Zeitgeist}

Die 1990er Jahre können aus heutiger Sicht als Hochzeit kommunaler Privatisierungspraxis von Infrastrukturunternehmen bezeichnet werden. Zunächst erfolgte die Absicherung der Netz- und Anlageinvestitionen kommunaler Ver- und Entsorger in Ostdeutschland in großem Umfang durch Privatkapital. Das war bundespolitisch befördert, wurde steuerlich begünstigt und durch unerfahrene, junge Kommunalpolitik in den fünf neuen Bundesländern umgesetzt. Ministerielle Task Forces und wie Pilze aus dem Boden geschossene Consultingunternehmen begleiteten diese Prozesse. Es fehlte hierfür jedoch vielfach schon am notwendigen prozessualen Know-How. Fehlgeplante überdimensionierte Anlagen, asymmetrische Risikolastverteilungen zuungunsten der öffentlichen Hand, erhebliche Transaktionskosten - derartige Befunde trugen erst Jahre später zu einer kritischen Bilanz dieser „Goldgräberjahre“ bei.

PPP war ein Zauberwort. Die Kooperation zwischen privaten Kapitaleignern und Kommunen sollte völlig neue Maßstäbe bei der kostengünstigen Erledigung öffentlicher Aufgaben setzen. Der erhebliche Nachholbedarf bei der Modernisierung von Wasser- und Abwasseranlagen, Stromnetzen und Kraftwerken, Verkehr und Entsorgung schien ohne Überlastung der unterfinanzierten kommunalen Haushalte realisierbar. PPP versprach schnelle und unkomplizierte Abhilfe zu beiderlei Vorteil.

Die Welle von Privatisierungen, Teilprivatisierungen und PPP in der kommunalen Ver- und Entsorgungsbranche griff schnell auch auf Kommunen im Westteil der Bundesrepublik über. Europäische Liberalisierungspolitik bei Dienstleistungen von allgemeinem wirtschaftlichen Interesse (DAWI), kommunale Finanznot, ein Überangebot anlagesuchenden privaten Investitionskapitals 
Die Teilprivatisierung der Berliner Wasserbetriebe: Erfolgsmodell oder Abwicklungsfall?

und ein derartigen „Entstaatlichungsprogrammen“ förderlicher Zeitgeist schufen ein Klima, in dem viele Kommunen sehr unbefangen und unkritisch ihr Tafelsilber zur Disposition stellten.

\section{Die Situation im Berlin der 1990er Jahre}

Auch Berlin erlebte in der 2. Hälfte der 1990er Jahre eine Privatisierungswelle. Dieser Verkauf von Daseinsvorsorgeunternehmen hatte jedoch noch einen ganz spezifischen Berliner Hintergrund: Nach dem Mauerfall stand nicht nur die infrastrukturelle Zusammenführung der geteilten Stadthälften an. Berlins sozioökonomische Basis war schwach, die Stadt verfügte kaum über eigene Potenziale zur Entwicklung einer wirtschaftlichen Perspektive. Ihre Industriestruktur war kaum im internationalen Maßstab wettbewerbsfähig; sie brach nach 1989/90 sukzessive weg. Dieser negativen Entwicklung standen ein aus „Frontstadtzeiten“ überkommener überdimensionierter Verwaltungsapparat und eine spezifische Verwaltungskultur gegenüber, die Berlin den Titel „Hauptstadt von Korruption und Filz“1 eintrug. Westberlin hatte vor dem Mauerfall aufgrund politischen Konsenses während der Teilung weitgehend als Subventionsmetropole, durch milliardenschwere Zuweisungen aus dem Haushalt des Bundes, gelebt. Die schrittweise Rückführung der Bundesmittel der „Berlinförderung“ bis 1995 bei weitgehend unveränderter Fortsetzung der Ausgabenpolitik des Landes ließen jährlich wachsende Etatlücken entstehen, die die Stadt absehbar in ihre fiskalische Handlungsunfähigkeit manövrieren mussten. ${ }^{2}$

Die schwarz-rote Koalition unter Eberhard Diepgen verständigte sich vor diesem Hintergrund 1995 auf eine Vermögensprivatisierungsstrategie. Der Verkauf der städtischen Energieunternehmen BEWAG und Gasag, In-sich-Geschäfte unter den städtischen Wohnungsbaugesellschaften und die Abtretung zukünftiger Dividendenansprüche aus der Beteiligung an der Berliner Bankgesellschaft/Landesbank sollten die jährliche Deckung von Einnahmen und Ausgaben im Landesetat sichern. Eine nachhaltige fiskalische Sanierungsstrategie der Stadtpolitik, mit Blick auf die Ausgabeseite, verband sich damit allerdings nicht. Stattdessen sollte ,aktivierende Stadtpolitik“ mittels öffentlicher Investitionen in Großprojekte quasi im Selbstlauf zur Entwicklung einer „,blühenden“ Dienstleistungsbasis führen. Berlin verharrte zwischen Größenwahn, Metropolenträumen und milliardenschweren Fehlinvestitionen, die den Fiskus der Stadt noch bis heute belasten.

In den Jahren 1997 und 1998 hatte Berlin mit Vermögensverkäufen jährlich ca. 6 Mrd. DM (ca. 3 Mrd. Euro) erlöst, die zur Schließung der immer stärker klaffenden Schere zwischen Einnahmen und Ausgaben aufgewandt worden sind. Für 1999 hatten der Senat und die Finanzsenatorin Fugmann-Heesing 3,4 Mrd. DM (ca. 1,7 Mrd. Euro) als Einnahme aus Vermögensprivatisierungen für den Landesetat geplant, für 2000 immerhin noch 2,4 Mrd. DM (ca. 1,2 Mrd. Euro). ${ }^{3}$ Die Einnahmeerzielung durch „Vermögensaktivierung“ beherrschte wie ein stummer Zwang des Faktischen die Berliner Landespolitik von 1995 bis zur Jahrtausendwende. Sie war die Geschäftsgrundlage der Koalition und ordnete sich sämtliche Sachüberlegungen zur Perspektive der Ber-

1 So der paradigmatische Titel des Buches von Rose (1997).

2 Ausführlich Weinzen (2000). Mit Stand 2001 hatte die Stadt einen Schuldenberg von 75 Mrd. DM (ca. 37 Mrd. Euro) angehäuft, bei einer jährlichen verbleibenden Deckungslücke von mehreren Mrd. DM.

3 Lederer (2010), S. 2. 
liner Daseinsvorsorge unter. So gerieten auch die BWB mit ihrem äußerst werthaltigen Anlagevermögen in den Fokus der „Vermögensaktivierung“‘.

\section{Der Prozess der BWB-Teilprivatisierung}

\section{Vorgeschichte}

\section{a) Vereinigung beider Stadthälften 1990 bis 1992}

Bis 1990 waren die wasserwirtschaftlichen Infrastrukturen Ost- und Westberlins organisatorisch und unternehmerisch getrennt. Erst 1988 waren im Westteil der Stadt die bislang eigenständigen Eigenbetriebe Wasserversorgung und Abwasserentsorgung in einem Betrieb verbunden worden. Mit dem Mauerfall 1989 und der Einheit Deutschlands 1990 stand die Stadt vor der Herausforderung, auch die netzgebundenen Wasserinfrastrukturen aus Ost und West zusammenzuführen. Aus dem Eigenbetrieb (West) und der VEB Wasser- und Abwasserbehandlung (WAB Ost) entstand 1992 ein gemeinsamer Eigenbetrieb. Mit der Zusammenführung der drei wasserwirtschaftlichen Entitäten entstand das größte Wasserver- und Abwasserentsorgungsunternehmen mit zusammenhängender Netz- und Anlagestruktur in Europa.

\section{b) Die Eigenbetriebsreform 1993: Bildung einer Anstalt öffentlichen Rechts}

Im Zuge der Debatten um eine Neuordnung der Ver- und Entsorgungsbetriebe Berlins entschied sich die schwarz-rote Koalition bald darauf zur Rechtsformänderung bei den Berliner Eigenbetrieben der Ver- und Entsorgungswirtschaft. ${ }^{4}$ Für Verkehrsbetriebe, Stadtreinigung und Wasserwirtschaft erließ das Abgeordnetenhaus von Berlin 1993 das Berliner Betriebegesetz. ${ }^{5}$ Aufgrund dessen wurde aus dem Sondervermögen des Eigenbetriebs BWB zum 1.1.1994 die Berliner Wasserbetriebe Anstalt öffentlichen Rechts (BWB AöR) errichtet.

Es entstand eine selbständige juristische Person BWB mit den Organen Vorstand, Aufsichtsrat und Gewährträgerversammlung. Der Vorstand der Anstalt ist zur eigenverantwortlichen Leitung und Rechtsvertretung der Anstalt befugt. Er wird vom Aufsichtsrat der AöR berufen und kontrolliert, der sich aus Landesvertretern und Vertretern der BWB-Beschäftigten zusammensetzt. Aufgabe der Gewährträgerversammlung war die Einsetzung und Kontrolle des Aufsichtsrats, die Wahrung der Interessen des Landes Berlin nach Maßgabe des Berliner Betriebegesetzes.

Da in Berlin Kommunalebene und Landesebene zusammenfallen, verfügt das Land - anders als Kommunen in den Flächenstaaten - über die besondere Möglichkeit, die gesetzlichen Rahmenbedingungen seiner Kommunalwirtschaft selbst gestalten zu können. Die Rechtsform der AöR

4 Zu den Hintergründen vgl. Gesellschaft für öffentliche Wirtschaft (1993).

5 Berliner Betriebegesetz (BerlBetrG) vom 9.7.1993, GVB1. S. 319. 
ist - anders als die der Kapitalgesellschaft beim Typenzwang des Gesellschaftsrechts - nicht spezifisch vorgegeben, sondern kann vom Gesetzgeber weitgehend frei gestaltet werden. ${ }^{6}$ Das Abgeordnetenhaus hat die Berliner Anstaltsstruktur ersichtlich an der kapitalgesellschaftlichen Unternehmensorganisation orientiert. Erklärtes politisches Ziel des Rechtsformwechsels war, den Unternehmensleitungen eine größere unternehmerische Selbständigkeit, eine größere Unabhängigkeit von politischer Steuerung und Einflussnahme, zu verschaffen. ${ }^{7}$ Abgesichert durch Anstaltslast und Gewährträgerhaftung des Landes Berlin sollten die Anstalten selbständig agieren und durch geeignete unternehmerische Expansionsstrategien die Entwicklung der Stadt zur Dienstleistungsmetropole befördern, Arbeitsplätze schaffen und private Investitionen an die Stadt binden. Die öffentlichen Unternehmen sollten „Cash-Maschinen“ sein. Im Gegenzug zu den gewährten Freiräumen durften die Unternehmensvorstände sich relativer Zurückhaltung seitens des Senats bei der Kontrolle ihrer Aktivitäten und Praktiken sicher sein.

\section{c) Auf Expansionskurs}

Die neuen Freiräume wurden vom Vorstand der BWB - mit Förderung und Billigung des Senats weidlich genutzt. $\S 2$ Abs. 7, 8 BerlBetrG gestattete den Anstalten, eigenverantwortlich Beteiligungen „im Rahmen ihrer allgemeinen Aufgabenstellung“ einzugehen. Die BWB entwickelten ein breites Beteiligungsportefeuille mit über 20 Tochtergesellschaften. ${ }^{8}$ Viele dieser Experimente erwiesen sich als wenig lukrativ und kamen Anstalt und Land schließlich teuer zu stehen.

Zwei „Flop“-Engagements der Wasserbetriebe aus dieser Phase stechen besonders hervor. Ihr Bezug zur ,allgemeinen Aufgabenstellung“ liegt erstens völlig im Dunkeln, zweitens summierten sich die versenkten Investitionsmittel hier auf exorbitante Höhen. Drittens fiel keine öffentliche Kontrollinstanz Berlins dem BWB-Vorstand in den Arm, um die Vernichtung von Mitteln der Tarifzahler bzw. von Steuergeldern aufzuhalten. Diese unternehmerischen Ausflüge der BWB in die Telekommunikations- und in die Entsorgungsbranche waren von keinerlei Sachkenntnis und Verantwortungsbewusstsein der BWB-Spitze getrübt:

Mit der Tochter BerliKomm versuchte der Vorstand der BWB, ein Unternehmen auf dem liberalisierten Telekommunikationsmarkt zu etablieren. Dazu sollten, so die Idee, die wasserwirtschaftlichen Netzstrukturen fruchtbar gemacht werden. Das ganze Unternehmen erwies sich schließlich als Millionengrab, das - nach der Teilprivatisierung - beendet werden musste. Mit ca. 220 Mio. Euro wurden die Kosten dieses Experiments beziffert. ${ }^{9}$ Mit der Übernahme des SVZ Schwarze Pumpe in Brandenburg, in dem Klärschlammverwertung mit Energieerzeugung gewinnbringend verbunden werden sollte, versuchten sich die BWB als Schrittmacher der Energieerzeugung aus Klärabfällen. Die zugrundeliegenden Technologien erwiesen sich aber als unausgereift für den industriellen Prozess. Als bei diesem Experiment 2002 die Notbremse gezogen

6 Vgl. Mann (1996).

7 Siehe hierzu die Gesetzesbegründung zum Eigenbetriebereformgesetz vom 9.7.1993 auf Abghs-Drs. 12/2897.

8 Überblick bei Senatsverwaltung für Finanzen (1999), S. 11. Es umfasste Unternehmen der Wassertechnik, des Consultings, aber auch zum Betrieb von Wasserinfrastrukturen in Osteuropa und Asien.

9 Schulte (2004). 
wurde, waren nicht nur größenwahnsinnige Träume zerplatzt, sondern ca. 620 Mio. Euro Verluste zulasten des Unternehmens und des Landeshaushalts aufgelaufen. ${ }^{10}$

\section{d) Zwischenfazit}

Der politische Wille der Regierungskoalition, der 1994 zur Anstaltsgründung führte, befreite die BWB von den engen öffentlich-rechtlichen Bindungen und der strikten Haushaltskontrolle eines Eigenbetriebs. Die unternehmerische Strategie, die der Vorstand der Berliner Wasserbetriebe daraufhin verfolgte, war durch Inkonsistenz und Voluntarismus gekennzeichnet. Neben dem „Kerngeschäft“, der Versorgung der Berliner Bevölkerung mit Wasser und der Entsorgung des im Stadtgebiet anfallenden Abwassers, etablierte die BWB-Spitze einen unüberschaubaren und nicht kontrollierbaren Wildwuchs von unternehmerischen Engagements. Der Senat ließ das zu, weil die in Aussicht gestellten Verheißungen der unternehmerischen Expansion der BWB ihm angesichts der klammen Kassenlage der Hauptstadt - zu verlockend erschienen.

Während die vorbildliche Qualität der Trinkwasserver- und Abwasserentsorgungsleistungen der BWB bundesweit hervorgehoben wurde, geriet das Unternehmen durch diese Geschäftspolitik insgesamt in Misskredit. Intransparenz, Missmanagement und Inkompetenz des BWB-Managements evozierten in der Öffentlichkeit zu Recht die Frage, welche Rechtfertigung es für diesen Umgang mit Landesmitteln und Tarifeinnahmen der Anstaltsnutzer geben könne.

\section{Die Teilprivatisierung der BWB 1998-1999}

\section{a) Erste Diskussionen}

Die schwarz-rote Koalition erwies sich als unfähig, aus den sich bereits 1997/1998 abzeichnenden Fehlentwicklungen der BWB die naheliegenden Schlüsse zu ziehen. Die Rückführung der Aufgaben der Anstalt auf das Kerngeschäft bei moderater Einnahmeerzielung für den Landeshaushalt, striktes Controlling der BWB-Wirtschaftstätigkeit, fachkundige Besetzung der Leitung und Sorgfalt in der unternehmerischen Führung - das hätte die Möglichkeit geboten, die BWB als stabiles Instrument der Stadtpolitik zu etablieren. Es fehlte der politische Wille, die Funktion der BWB für das Land neu zu justieren und eine adäquate Corporate Governance zu entwickeln und durchzusetzen. Die Koalition war in den Strategien des Metropolenwahns und der „Vermögensaktivierung" aus fiskalischer Motivation gefangen.

Als der Senat 1997 an die Veräußerung der Gasag-Anteile ging, brachte der BWB-Vorstandsvorsitzende Bertram Wieczorek mit Verweis auf die komfortable Eigenkapitalquote der Anstalt die Übernahme der Gasag durch die BWB ins Gespräch. Das ermunterte den Berliner Senat zur Entnahme von 1 Mrd. DM (ca. 500 Mio. Euro) aus dem Eigenkapital der BWB für die Haushaltskonsolidierung. Die Gasag-Anteile wurde dagegen 1998 (wie bereits 1997 die der BEWAG) von privaten Energieversorgungsunternehmen übernommen. Angesichts der hohen Deckungslü-

10 Schulte (2002). 
cken, die die mittelfristigen Finanzprognosen für den Landesetat auch in den bevorstehenden Haushaltsjahren offenbarten, drohte die Strategie der „Vermögensaktivierung“ 1997/1998 an ihre Grenze zu geraten. Nun begann alles auf die „Vermögensaktivierung“ des letzten „werthaltigen“ Versorgungsunternehmens, der BWB, hinauszulaufen. Bereits 1997 diskutierte die Koalition intern über die Perspektiven der BWB-Entwicklung. Dabei wurden auch Überlegungen über eine Privatisierung der BWB angestellt. ${ }^{11}$

Finanzsenatorin Fugmann-Heesing hatte für den Landeshaushalt des Jahres 1998 erneut Einnahmen aus Vermögensverkäufen in Milliardenhöhe veranschlagt, die noch nicht mit konkreten Vermögensveräußerungen untersetzt waren. Es war auch realistisch, bei einem Verkauf der BWB auf das Interesse kapitalkräftiger Bieter zu treffen. Sowohl internationale Finanz- als auch Branchenakteure waren für einen „Einstieg“ in das „Wassergeschäft“ der deutschen Hauptstadt mobilisierbar. ${ }^{12}$ Die Übernahme des größten siedlungswasserwirtschaftlichen Versorgungsgebiets in der Mitte Europas versprach neben einer lukrativen Kapitalanlage das Renommee, das gute Referenzen für weitere internationale Beteiligungen und damit eine solide Ausgangsbasis für die Expansion im globalen Wassermarkt bot. Ein strategisches Investment internationaler Wasserkonzerne ließ daher die Realisierung eines hohen Kaufpreises erwarten.

Angesichts der „fiskalischen Lücke“ war Eile geboten. Außerdem standen im Herbst 1999 Wahlen zum Abgeordnetenhaus an. Die Abwicklung eines Projekts dieses Umfangs und von einer solchen Tragweite war ein sehr ehrgeiziges Ziel. Nennenswerte Widerstände gegen eine Privatisierung der BWB waren im Berliner Landesparlament zwar nicht zu erwarten - die Schließung von Haushaltslücken durch Privatisierung von Landesunternehmen entsprach dem Konsens der Koalition. Die Frage war aber, wie das Projekt zu bewerkstelligen wäre und in der Stadtgesellschaft, insbesondere bei den Beschäftigten der Wasserbetriebe und der in der politischen Kultur Berlins traditionell einflussreichen Gewerkschaft ÖTV, legitimiert werden konnte.

\section{b) Privatisierungsvorbereitungen}

In der koalitionsinternen Verständigung gab es zunächst keine grundlegenden politischen Vorbehalte bezüglich der Rechtsformwahl und des Privatisierungsumfangs. Klar war nur, dass Betriebsführungs- und Betreibermodelle ausschieden. Sie ermöglichten keine Vermögensverschiebung mit entsprechenden Erlösen für den Landesetat, sondern erschlossen bestenfalls kontinuierliche Einnahmen auf vergleichsweise niedrigem Niveau über lange Zeiträume. Dennoch legten sich Senat und Koalition alsbald auf ein Privatisierungsmodell fest, das sowohl durchsetzbar erschien als auch adäquate Verkaufserlöse in Aussicht stellte: Die BWB sollten als Anstalt öffentlichen Rechts erhalten bleiben und im Rahmen einer Konzernierung in einen privatrechtlichen Konzern eingegliedert werden. Gegenüber der Umwandlung der BWB in eine Kapitalgesellschaft und ihrer Vollprivatisierung hatte das viele Vorteile: ${ }^{13}$

11 Vgl. Ochmann (2005), S. 21.

12 Zu dieser Zeit wurde international über die Geschäftsaussichten mit dem „,blauen Gold“ intensiv diskutiert. Zur ökonomischen Sicht auf die Wettbewerbstauglichkeit des ,natürlichen Monopols“ Wasserversorgung vgl. Stuchtey (2002); kritisch aus verwaltungswissenschaftlicher Perspektive zur Wasserver- und Abwasserentsorgung Lederer (2004), S. $407 \mathrm{ff}$.

13 Hierzu auch Ochmann (2005), S. 27 ff.; ferner Hüesker (2011), S. 120 ff. 
Die Errichtung der BWB AöR 1994 war mit einem Wechsel der BWB-Beschäftigten aus dem Arbeitsverhältnis mit dem Land in die AöR verbunden. Der Rechtsformwechsel fand die Zustimmung von Beschäftigten und Gewerkschaft nur, weil damit ein gesetzlicher Anspruch gegenüber dem Land Berlin in $§ 14$ Abs. 6 BerlBetrG verbunden wurde, bei erneutem Rechtsformwechsel wieder zum Land zurückkehren zu können. Die Inanspruchnahme dieses Rechts durch verunsicherte Beschäftigte erschien der Koalition fiskalisch als zu riskant. Mit der Erhaltung der Anstalt stellte sich das Problem nicht. Die ÖTV postulierte zwar prinzipiell die Ablehnung der BWBPrivatisierung, zeigte sich aber bereit, bei Wahrung der Interessen der Kernbelegschaft auf eine grundsätzliche Blockadehaltung zu verzichten. ${ }^{14}$

Traditionell wird in der Bundesrepublik Abwasserentsorgung als hoheitliche Tätigkeit begriffen, während Wasserversorgung als wirtschaftliche Tätigkeit gilt. ${ }^{15}$ Die kommunale Abwasserentsorgung in öffentlich-rechtlicher Rechtsform wird damit korrespondierend steuerrechtlich nicht als „Betrieb gewerblicher Art“ nach $\S \S 1$ Abs. 6, 4 KStG betrachtet, sondern als Hoheitsbetrieb. Das damit verbundene Steuerprivileg entfällt aber, sobald die Abwasserentsorgung in einer kommunalen Kapitalgesellschaft erbracht wird. Eine Sicherung der öffentlich-rechtlichen Rechtsform erhielt also monetäre Spielräume für preiswirksame Maßnahmen oder für die Gewinnerzielung. Schließlich verfügte das Land über einschlägige Erfahrungen mit der Anstaltskonzernierung. Bereits 1994 war die Landesbank Berlin AöR im Rahmen ihrer Teilprivatisierung in die Berliner Bankgesellschaft AG eingegliedert worden, ${ }^{16}$ wodurch sich die Vorteile von Anstaltslast und Gewährträgerhaftung unmittelbar kapitalisieren ließen: Die insolvenzunfähige öffentliche Hand steht für Verluste aus dem Geschäft unbegrenzt ein, was eine günstige Kapitalaufnahme ermöglicht. ${ }^{17}$ Der Rückgriff auf ein Modell „geübter Praxis“ ließ ferner schwächere Vorbehalte im politischen und stadtgesellschaftlichen Raum befürchten.

Am 7. Juli 1998 beschloss der Senat von Berlin die Teilprivatisierung in Form eines Holdingmodells unter Wahrung des Anstaltscharakters der BWB. Ein halbes Jahr später, am 5. Januar 1999, folgte ein Senatsbeschluss, der die Grundzüge des späteren Privatisierungskonstrukts bereits vorwegnahm und den Gesetzgebungsprozess im Berliner Abgeordnetenhaus in Gang setzte. ${ }^{18}$ Kern der vorgesehenen strukturellen Änderung der BWB-Unternehmensverfassung war die Befugnis der Anstalt, im Rahmen eines Unternehmensvertrages ihre Leitung einer juristischen Person des privaten Rechts zu unterstellen, wenn das Land Berlin an dieser mehrheitlich beteiligt und der Einfluss des Landes bei der Erteilung von Weisungen gewährleistet ist. ${ }^{19}$ Damit sollte die gesetzliche Voraussetzung zur Einbeziehung der Anstalt in einen privatrechtlichen Konzern geschaffen werden, die eine atypisch stille Beteiligung Privater an der öffentlich-rechtlichen Anstalt ermöglichte.

14 Mit Erfolg: Das Land kam der ÖTV im Privatisierungsverfahren noch weiter entgegen. Es sicherte den BWBBeschäftigten den Verzicht auf betriebsbedingte Kündigungen und Besitzstandswahrung bis $2014 \mathrm{zu}$.

15 Lederer (2004), S. 239 ff.

16 Zur Anstaltskonzernierung Bezzenberger/Schuster (1996); insbesondere zum „Berliner Modell“ Fett (2000).

17 Hier lag letztlich die Ursache für die Milliardenlasten Berlins aus dem „Berliner Bankenskandal“ 2001. Wenngleich diese Einstandspflicht Berlins im Fall der BWB nicht vordergründig relevant war, verbinden sich mit der Insolvenzunfähigkeit auch in diesem Fall finanziell messbare Vorteile; siehe Ochmann (2005), S. 31.

18 Vorlage - zur Beschlussfassung - über Gesetz zur Änderung des Berliner Betriebegesetzes, zur Teilprivatisierung der Berliner Wasserbetriebe und zur Änderung des Berliner Wassergesetzes vom 5. Januar 1999, AbghsDrs. $13 / 3367$.

19 Ebd., Art. II $\S 1$ Abs. 2, S. 2 und 3 im Senatsentwurf des Artikelgesetzes. 


\section{c) Beschlussfassung über das Teilprivatisierungsgesetz}

Die Interessenbekundung, die Auswahl der möglichen Investoren und die Verhandlungen zu den Teilprivatisierungsverträgen liefen parallel zum parlamentarischen Gesetzgebungsverfahren. Am 14. Januar 1999 fand die erste Lesung des Gesetzentwurfs im Landesparlament statt. Schon am 15. Januar 1999 lief jedoch die Frist aus, innerhalb derer potenzielle Bieter auf Grundlage eines Memorandums der (vom Land Berlin mit der Investorensuche beauftragten) Investmentbank Merrill Lynch erste Angebote abgeben konnten.

Beide Verfahren, sowohl das Gesetzgebungsverfahren als auch die Vertragsaushandlung, wurden vom Senat gesteuert und auf parlamentarischer Ebene von der Koalitionsmehrheit abgesichert. Die Erarbeitung der konkreten Gesetz- und Vertragsentwürfe lag aber in der Hand von hochspezialisierten Anwaltskanzleien, deren Repräsentanten auch in den parlamentarischen Ausschussberatungen Stellungnahme bezogen. ${ }^{20}$

Aufschluss über die parlamentarischen Beratungen vermitteln die Verhandlungen im Ausschuss für Wirtschaft und Betriebe des Berliner Abgeordnetenhauses. ${ }^{21}$ Die Abgeordneten befassten sich mit den verfassungsrechtlichen, tarifrechtlichen und ökonomischen Aspekten des vorgesehenen Teilprivatisierungsprojekts. Die Beschäftigungs- und Ausschreibungspolitik der BWB, Wasserpreis- und Gewinnentwicklung, der verbleibende Einfluss des Landes Berlin und die Haftung für Risiken, die Sicherung der ökonomischen Stabilität der BWB, die Perspektiven der BWB-Töchter wurden diskutiert. Die Kritik der Opposition galt der Intransparenz und Geschwindigkeit des Verfahrens. Immer wieder wurde hervorgehoben, dass die Details der parallel laufenden Vertragsverhandlungen den Abgeordneten nicht bekannt seien. Das Beratungsverfahren ähnelte daher in den verzwickten Detailfragen der Untersuchung einer Blackbox.

Die Debatten offenbarten aber auch handwerkliche Schwächen des Gesetzentwurfs. Diese korrigierte ein am 29. März 1999 angenommener Änderungsantrag der Koalition. ${ }^{22}$ Auf grundlegende Änderungen des Privatisierungsmodells und des Gesetzentwurfs wurde verzichtet. Bedenken über bevorstehende Anhebungen des Wasserpreises begegnete die Regierungskoalition mit der Festlegung des Tarifs auf dem Niveau bei Inkrafttreten des Gesetzes bis einschließlich 31. Dezember 2003. Sämtliche Anträge der Opposition ${ }^{23}$ lehnte die schwarz-rote Ausschussmehrheit ab. ${ }^{24}$ In der gleichen Sitzung legten die Regierungsfraktionen als Tischvorlage einen Antrag auf Entschließung ${ }^{25}$ vor, der den Senat in Hinblick auf die Verhandlungen mit möglichen Bietern zur Durchsetzung u. a. folgender Parameter aufforderte: Sicherung der Beschäftigtenrechte und Gewährleistung betrieblicher Mitbestimmung, Sicherung der wirtschaftlichen Unabhängigkeit und des Sitzes der BWB in Berlin, Beteiligung der Kunden und Beschäftigten durch Aktienausgabe, Einrichtung eines mehrheitlich durch das Land Berlin besetzten Weisungsausschusses zur Be-

20 Zum zeitlichen Ablauf Hüesker (2011), S. $139 \mathrm{ff}$.

21 Vgl. hierzu die Ausschussprotokolle 13/32 der 32. Sitzung vom 25.1.1999 und 13/36 der 36. Sitzung vom 29.3.1999.

22 Abghs-Drs. 13/3367; siehe Anlage 1 zum Beschlussprotokoll der 36. Sitzung des Ausschusses für Wirtschaft und Betriebe des Abgeordnetenhauses von Berlin am 29.3.1999.

23 Abghs-Drs. 13/3608 sowie 13/3614-3616.

24 Vgl. Beschlussprotokoll der Ausschusssitzung, a.a.O. So forderten die Grünen, auf das Holdingmodell zugunsten eines Konzessionsmodells zu verzichten. Das Senatsmodell wurde von den Fraktionen der PDS und der Grünen abgelehnt.

25 Abghs-Drs. 13/3618; vgl. Anlage 2 zum Beschlussprotokoll, a.a.O. 
stätigung von Weisungen der herrschenden Gesellschaft gegenüber der Anstalt, Sicherstellung, dass 50,1 Prozent der Anteile an der herrschenden Gesellschaft (Holding-AG) auch zukünftig beim Land Berlin verbleiben. 10 Prozent des Erlöses der Teilprivatisierung sollten in einen nicht näher bezeichneten „Zukunftsfonds“ eingebracht werden.

Das gleiche Geschehen wiederholte sich in der entscheidenden Sitzung des Hauptausschusses des Abgeordnetenhauses ${ }^{26}$ vor der 2. Lesung des Gesetzes im Parlament am darauffolgenden Tag. Auf seiner 62. Sitzung am 29. April 1999 beschloss das Abgeordnetenhaus von Berlin das Gesetz zur Änderung des Berliner Betriebegesetzes, zur Teilprivatisierung der Berliner Wasserbetriebe und des Berliner Wassergesetzes ${ }^{27}$ und die von SPD und CDU vorgelegte begleitende Parlamentsentschließung.

\section{d) Die Entscheidung zum Vermögensgeschäft}

Senatsseitig war das Verkaufsverfahren inzwischen bis in die entscheidende Runde vorangetrieben. Am 18. Juni 1999 erteilte der Senat einem Konsortium aus RWE, Vivendi ${ }^{28}$ und Allianz für die Übernahme von 49,9 Prozent an den Wasserbetrieben den Zuschlag. Das Konsortium hatte mit einem Kaufpreis von 3,4 Mrd. DM (ca. 1,7 Mrd. Euro) das bei Weitem höchste Angebot abgegeben ${ }^{29}$ und sich zur Übernahme weiterer Verpflichtungen bereiterklärt. ${ }^{30}$ Die Laufzeit des Vertrags sollte 30 Jahre betragen, erstmalig regulär kündbar mit einer Frist von fünf Jahren vor Ablauf. Über den Inhalt der Verträge zur Realisierung der Teilprivatisierung vereinbarten die beteiligten Vertragspartner die Wahrung der Vertraulichkeit. Neben der Kaufpreishöhe waren weitere Aspekte maßgeblich: Von der Einbeziehung des französischen Wasserkonzerns Vivendi/ Veolia versprach sich der Senat einen Ausbau der BWB-Beteiligungen. Aus Sicht von Veolia war das Konsortium mit RWE eine vertrauensbildende Maßnahme gegenüber Berlin, um sich mit dem Einstieg bei den BWB den Ausbau der eigenen Stellung in Deutschland und Osteuropa zu sichern. ${ }^{31}$ RWE hatte begonnen, eine globale Expansionsstrategie im Wassersektor zu entwickeln, die aber später wieder aufgegeben wurde. Dem Abgeordnetenhaus und der Öffentlichkeit wurden sinkende Wasserpreise infolge der Veräußerung in Aussicht gestellt.

Mit der Auswahl des Partners ging das Vermögensgeschäft in die Schlussphase. Am 28. Juni 1999 befasste sich der Wirtschaftsausschuss, dem die „Eckpunkte“ der Senatsentscheidung vorgestellt wurden, mit den „Auswirkungen der Teilprivatisierung der BWB“. ${ }^{32}$ Die dem Geschäft zugrundeliegenden Verträge wurden allerdings nur im nicht-öffentlich tagenden Vermögensausschuss

26 Hierzu Ausschussprotokoll 13/88 der Sitzung des Hauptausschusses am 30.3.1999.

27 GVB1., S. 183, vom 17.5.1999.

28 Heute (und im Folgenden im Text): Veolia.

29 Davon wurden 3,1 Mrd. Euro für das Land Berlin kassenwirksam, während die restliche Summe zur ,Altlastensanierung“, etwa zur Lastentragung für das SVZ Schwarze Pumpe, aufgewendet wurde. S. dazu bereits oben, II. 1. c).

30 Dazu gehörten u. a. eine Verpflichtung zu Schaffung von Arbeitsplätzen und eines Wasserforschungszentrums, die Sicherung der Beschäftigungsgarantie bis 2014, eine Investitionsverpflichtung der Wasserbetriebe in Höhe von 10 Mrd. DM (ca. 5 Mrd. Euro) bis zum Jahr 2009.

31 Der dritte Partner Allianz diente als Finanzier und zog sich später planmäßig aus dem Engagement zurück.

32 Vgl. das Inhaltsprotokoll 13/41 der 41. Sitzung des Ausschusses für Wirtschaft und Betriebe des Abgeordnetenhauses Berlin vom 28.6.1999. 
behandelt, der dem Plenum die Zustimmung zum Vermögensgeschäft empfahl. Am 1. Juli 1999 stimmte das Abgeordnetenhaus mit der Mehrheit der Koalitionsfraktionen dem Vermögensgeschäft zu. ${ }^{33}$ Daraufhin wurden die Teilprivatisierungsverträge unterzeichnet.

\section{e) Struktur des „BWB-Konzerns“}

Zur Realisierung der atypisch stillen Beteiligung an der BWB war ein umfangreiches Vertragswerk ausgehandelt worden. ${ }^{34}$ Zunächst gründet das Land eine Holding-AG, auf die das „Wettbewerbsgeschäft“ der BWB AöR übertragen wird. Eine zu diesem Zweck gegründete Beteiligungs-AG der privaten Anteilseigner übernimmt 49,9 Prozent der Aktien der Holding-AG, das Land Berlin behält die Aktienmehrheit von 50,1 Prozent. Durch einen ersten stillen Gesellschaftsvertrag beteiligt sich die Holding-AG gegen Einbringung einer Einlage in Höhe von 49,9 Prozent des BWB-Vermögens schuldrechtlich am Kapital der BWB AöR. Mittels eines weiteren stillen Gesellschaftsvertrags zwischen Holding-AG und Beteiligungs-AG übernehmen die privaten Anteilseigner die Einlage aus der schuldrechtliche Beteiligung der Holding-AG an den BWB AöR vollständig. Das Land Berlin kann nun in gleicher Höhe Eigenkapital aus den BWB AöR entnehmen, womit wirtschaftlich die Realisierung des „Kaufpreises“ durch RWE/Veolia/Allianz umgesetzt ist.

Aufgrund des Vertrags über die Begründung einer einheitlichen Leitung zwischen Holding-AG und BWB AöR wird der Holding ein Weisungsrecht gegenüber der Anstalt eingeräumt. Das Weisungsrecht ist in seinem Inhalt durch das Teilprivatisierungsgesetz rechtlich beschränkt und steht unter dem Vorbehalt der Zustimmung durch den mehrheitlich vom Land besetzten Weisungsausschuss. Ein Interessenwahrungsvertrag zwischen dem Land Berlin und der Holding-AG konkretisiert das Verfahren der Besetzung der von der Gewährträgerversammlung der BWB AöR zu bestellenden Aufsichtsratsmitglieder: Der Holding wird das Recht eingeräumt, dem Land Berlin Vorschläge für die der Gewährträgerversammlung zur Bestellung vorzuschlagenden Aufsichtsratsmitglieder zu unterbreiten. Dazu wird dem Land durch die Holding-AG eine Liste mit drei Vorschlägen für jedes vom Land zu besetzende Aufsichtsratsmandat zugeleitet, aus dem der Senat auswählen kann. Gegebenenfalls ist der Senat berechtigt, eine Listenergänzung zu verlangen. Das Land verpflichtet sich also mit diesem Vertrag im Verhältnis zur Holding-AG, in Wahrnehmung seines Vorschlagsrechts gegenüber der BWB-Gewährträgerversammlung für die vom Land zu besetzenden Aufsichtsratsmitglieder der BWB nur solche Personen vorzuschlagen, die auf einen Vorschlag der Holding-AG zurückgehen.

33 Plenarprotokoll 13/66 der 66. Sitzung des Abgeordnetenhauses vom 1.7.1999 (Anlage 2, Geschäft Nr. $17 / 1999$ des Verzeichnisses über Vermögensgeschäfte).

34 Vgl. hierzu ausführlich Hecker (2001), S. 265 ff.; Ochmann (2005), S. 38 ff.; Hüesker (2011), S. 142 ff. Die Verträge sind inzwischen veröffentlicht. Grundlage war eine Novellierung des Berliner Informationsfreiheitsgesetzes (IFG) im Juli 2010 (vgl. Abghs-Drs. 16/3340 vom 28.6.2010), die einen Verhandlungsmechanismus zur Vertragsoffenlegung eingeführt hatte. RWE und Veolia stimmten daraufhin im November 2010 der Offenlegung zu. Durch Volksentscheid wurde am 13.2.2011 ein weiteres Gesetz zur Vertragsoffenlegungspflicht beschlossen, das aber in dieser Hinsicht keinen praktischen Anwendungsbereich mehr fand. 


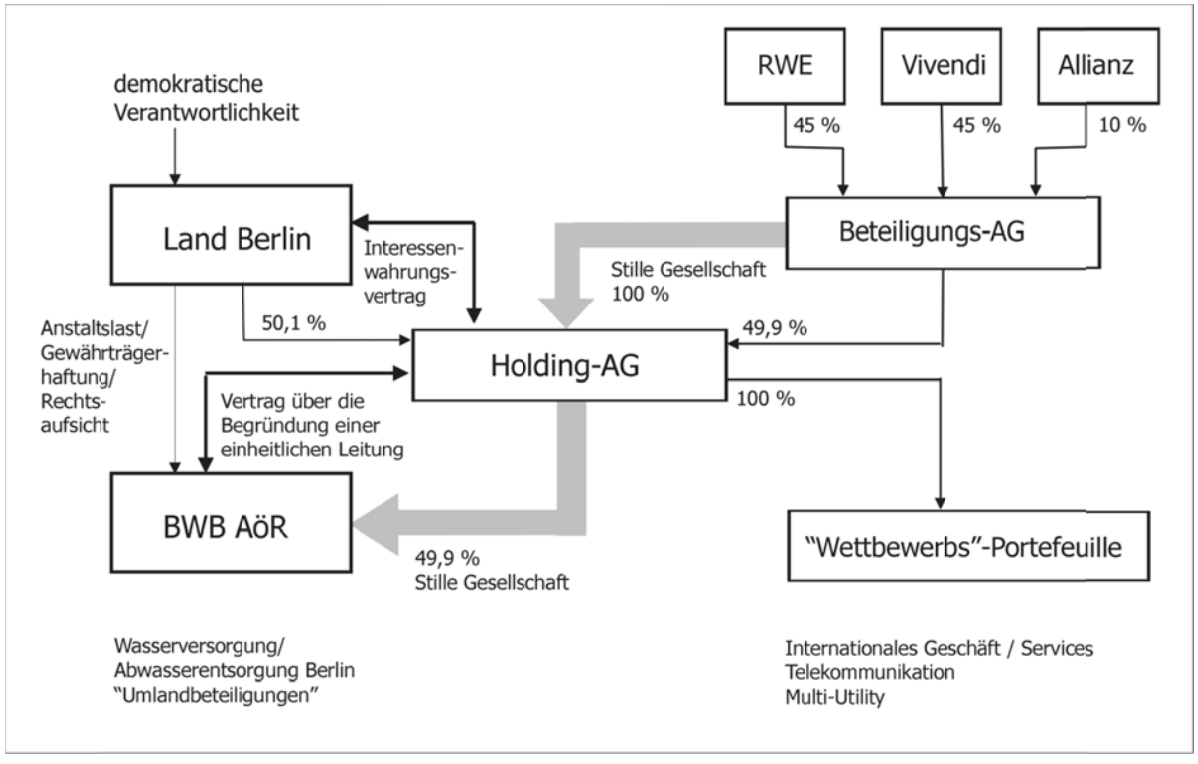

Abbildung 1: Holdingmodell zur Konzernierung der BWB AöR

Quelle: Lederer (2004), S. 344

Die Rahmenvereinbarung zur Realisierung der Teilprivatisierung, abgeschlossen zwischen allen Beteiligten, bildet der Konsortialvertrag. Er beinhaltet die maßgeblichen Absprachen zu den geschäftlichen Zielen des Landes und der privaten Anteilseigner, konkretisiert die Regelungen zur Besetzung von Aufsichtsrat und Vorstand der Holding-AG und der BWB AöR, enthält Regelungen zu den Rechten und Pflichten der Investoren, zu den Gewinnansprüchen und zur Verteilung der Gewinne.

\section{f) Zwischenfazit}

Der Verlauf der Plenar- und Ausschussberatungen zeigt, wie immens der durch die Koalitionspolitik auferlegte Zeitdruck war. ${ }^{35}$ Das begrenzte die Möglichkeiten der Abgeordneten, sich sachkundig in die komplexe Rechtsmaterie einzuarbeiten und die tatsächlichen Auswirkungen des Projekts zu diskutieren. Letztlich standen grundsätzliche Alternativen zum (oder auch nur relevante punktuelle Veränderungen am) Holdingmodell mit Aussicht auf Realisierung im Parlament niemals ernsthaft zur Debatte. Die normative Kraft des vom Senat in Gang gesetzten faktischen Verfahrens verengte den Spielraum der regierungstragenden Parlamentsmehrheit, die sich dem auch nicht entzog. Die schließlich von der Koalitionsmehrheit beschlossene Entschließung zur

35 Die Erlöse aus dem geplanten Vermögensgeschäft waren bereits zur Deckung von Einnahmeerwartungen für das zurückliegende Haushaltsjahr 1998 vorgesehen. Das erforderte den Kaufpreisfluss bis zur Schließung der Bücher zum 31.12.1999; vgl. Plenarprotokoll der 62. Sitzung des Abgeordnetenhauses am 29.4.1999, S. 4511 ff. 
Umsetzung der Teilprivatisierung zeigt, dass sich auch Teile der Regierungsfraktionen unsicher und der Tragweite der Maßnahme nicht in voller Konsequenz bewusst waren, als sie das Teilprivatisierungsgesetz verabschiedeten. Bei der Abstimmung über das Privatisierungsgesetz gab es 7 Nein-Stimmen und 11 Enthaltungen aus dem Koalitionslager. Über das Vermögensgeschäft entschied die große Mehrheit der Abgeordneten ohne Kenntnis der konkreten Vertragsabreden und ihrer Konsequenzen.

\section{Vollzug der Teilprivatisierung und Praxis der BWB-Steuerung}

\section{Das Verfassungsgerichtsverfahren der Opposition}

\section{a) Das Urteil des Verfassungsgerichts vom 21. Oktober 1999}

Der beschlossenen Teilprivatisierung stand, nachdem alle parlamentarischen Hürden genommen waren, nur noch ein Hindernis im Weg: Die überstimmte Parlamentsopposition aus Grünen und PDS hatte gegen das Teilprivatisierungsgesetz einen abstrakten Normenkontrollantrag vor dem Verfassungsgerichtshof des Landes Berlin erhoben. Bis zum Abschluss dieses Verfahrens wurde der Vollzug des Geschäfts ausgesetzt.

Die Oppositionsabgeordneten beanstandeten erstens, dass die gewählte Konstruktion - Einräumung mitunternehmerischer Rechte an eine privatrechtliche Gesellschaft durch atypisch stille Beteiligung am Anstaltsvermögen - gegen das Demokratieprinzip verstoße. Die Aufgabenerfüllung einer Anstalt öffentlichen Rechts sei Staatstätigkeit, die einer demokratischen Legitimation bedürfe. Die Unterstellung der Anstalt unter die Leitungsmacht einer privatrechtlichen Holding sei damit nicht vereinbar. Der Zurechnungszusammenhang zwischen Parlament und Anstalt sei auch durch das Verfahren zur Aufsichtsratsbesetzung der BWB unterbrochen.

Zweitens griffen die Antragsteller Regelungen des Teilprivatisierungsgesetzes an, die als Grundlage für die Tarifbemessung eingeführt worden sind. Die Tarife sollten nach dem Teilprivatisierungsgesetz kostendeckend festgesetzt werden. Zur Bemessung stellt das Gesetz auf die bei wirtschaftlicher Betriebsführung nach betriebswirtschaftlichen Grundsätzen ansatzfähigen Kosten ab. Neben den tatsächlichen Aufwendungen sind daher auch fiktive Kostenpositionen einzubeziehen. Diese fiktiven Kosten sollten eine angemessene kalkulatorische Verzinsung des betriebsnotwendigen Anstaltskapitals sichern. Als angemessene kalkulatorische Verzinsung war nach $\S 3$ Abs. 4 des Teilprivatisierungsgesetzes die durchschnittliche Rendite zehnjähriger deutscher Bundesanleihen in einem Zeitraum von 20 Jahren vor der jeweiligen Kalkulationsperiode (,Referenzzinssatz") zuzüglich 2 Prozentpunkte zugrunde zu legen (,R+2-Regelung“). Schließlich sollte auch eine darüber hinausgehende Verzinsung als angemessen gelten, sofern sie auf Maßnahmen beruht, die zu einer dauerhaften Steigerung der Wirtschaftlichkeit des Anstaltsbetriebes führen („Effizienzsteigerungsklausel“), dies allerdings nur für die Dauer von 3 Jahren - ab dem vierten Jahr waren die erzielten Kostensenkungen durch Entgeltreduzierung an die Wasserkunden weiterzugeben. Beide Regelungen waren im Rahmen des Teilprivatisierungsverfahrens entwickelt worden. Sie dienten der Sicherung der Gewinnerwartungen der Anteilseigner und der Etablierung 
von Effektivitätsanreizen zum unmittelbaren Vorteil von Land und Konsortium bei der unternehmerischen Führung der BWB. In beiden Vorschriften sahen die Antragsteller Instrumente der Gewinnmaximierung und einen Verstoß gegen haushaltsverfassungsrechtliche Grundsätze.

Der Verfassungsgerichtshof des Landes Berlin folgte den Antragstellern nur in Bezug auf die tarifrechtlichen Sonderregeln, im Übrigen wurde der Normenkontrollantrag zurückgewiesen. ${ }^{36}$ Die Anstaltskonzernierung der BWB wurde in verfassungskonformer Auslegung als zulässig erachtet, weil die Erteilung von Weisungen der Holding gegenüber der Anstalt aufgrund der gesetzlichen Anordnung in $\S 1$ Teilprivatisierungsgesetz ihrer ordnungsgemäßen Aufgabenerfüllung, Anstaltslast, Gewährträgerhaftung und Anstaltsträgerschaft nicht zuwiderlaufen dürfen. Außerdem sei durch die Etablierung einer Rechtsaufsicht und der Zustimmungspflicht eines Weisungsausschusses aus mehrheitlich vom Land Berlin entsandten Vertretern die demokratische Legitimation gesichert - jedenfalls mit der Maßgabe, dass zur Sicherung des notwendigen Legitimationsniveaus bei den Entscheidungen des Ausschusses das Prinzip der doppelten Mehrheit gesichert sei. Das Verfassungsgericht fordert zur parlamentarischen Rückbindung allerdings, dass allen Abgeordneten Einsicht in die Verträge gewährt werden muss. ${ }^{37}$ Auch die Besetzung des Aufsichtsrats der BWB auf Grundlage einer Vorschlagsliste der Holding wurde mit Verweis auf das Besetzungsverfahren für ehrenamtliche Richter für verfassungskonform gehalten.

Die tarifrechtlichen Besonderheiten zur Absicherung des von den Anteilseignern erwarteten Gewinns, nämlich die „R+2-Regelung“ und die „Effizienzsteigerungsklausel“, erklärte das Gericht für verfassungswidrig und nichtig. Beide Vorschriften stünden im Widerspruch zum Verhältnismäßigkeitsprinzip, das eine sachgerechte Verknüpfung zwischen den Kosten und der Höhe des zu erhebenden Entgelts fordert. Bei den genannten Regelungen sei die verfassungsrechtlich gebotene sachliche Rechtfertigung für ihre Einbeziehung in die Tarifkalkulation nicht erkennbar. Die Vorgabe der Gewinnerzielung für die Anstalt an sich beanstandete das Gericht aber nicht.

\section{b) Vertragliche Absicherung der Investoren: Nachteilsausgleichspflicht Berlins}

Damit war das „Holdingmodell“ für grundsätzlich verfassungsrechtlich zulässig erklärt worden. Die Teilprivatisierung wurde daraufhin am 29. Oktober 1999 vollzogen. ${ }^{38}$ Mittels seiner schuldrechtlichen Beteiligung ,kapitalisierte“ das Konsortium über die Holding die BWB AöR, während das Land den „Kaufpreis“ aus dem Eigenkapital der BWB AöR entnahm. Er floss der Landeskasse zu. Damit fand das wertmäßig größte Geschäft der Berliner Ära der „Vermögensaktivierung“ seinen Abschluss.

Problematisch erschien aus der Perspektive von Senat und Privaten allerdings die Nichtigerklärung der beanstandeten tarifrechtlichen Vorschriften. Sie dienten beiden Seiten bei der langfris-

$36 \mathrm{Zu}$ den Einzelheiten vgl. BerlVerfGH 42/99, Urteil vom 21.10.1999.

37 Zustimmend im Ergebnis Wolfers (2000); Schmehl (2001), S. 235, und wohl auch Schwintowski (2001); zweifelnd im Ergebnis Hecker (2001). Ochmann (2005) sieht dagegen beim „Holdingmodell“ die Anforderungen aus Art. 20 Abs. 2 GG ganz klar nicht gewahrt, bezieht aber die konkrete vertraglich ausgehandelte Kompetenzverteilung zwischen Land und Investoren mit in ihre Betrachtungen ein. Der Verfassungsgerichtshof hat sich zuständigkeitsgemäß auf die Normenkontrolle des Teilprivatisierungsgesetzes beschränkt.

38 In einer daraufhin von PDS und Grünen herbeigeführten Sondersitzung des Abgeordnetenhauses am 26.10.1999 wurde ein Oppositionsantrag auf Neuverhandlung der Verträge (Abghs-Drs. 13/4170) abgelehnt und der Senat zum Vollzug der Teilprivatisierung aufgefordert. 
tigen Realisierung ihrer Gewinnerwartungen und waren daher für den Erfolg der BWB-Privatisierung entscheidend.

Der Senat und die privaten Partner hatten jedoch in Kenntnis des laufenden Normenkontrollverfahrens der Opposition bereits vorab vertragliche Vorsorge getroffen. $§ 23$ Abs. 7 des Konsortialvertrags nahm für die aus einer möglichen negativen Verfassungsgerichtsentscheidung resultierenden Gewinneinbußen der privaten Anteilseigner unmittelbar das Land Berlin und damit den Landeshaushalt in die Haftung: Das Land verpflichtete sich, in einem solchen Fall zunächst alle legalen und wirtschaftlichen Maßnahmen ergreifen, um den BWB Gewinnausschüttungen in gleicher Höhe zu ermöglichen, wie sie bei Fortgeltung der für nichtig erklärten Kalkulationsvorschriften realisierbar gewesen wären. Sollten solche Maßnahmen unmöglich sein, verpflichtete sich das Land Berlin RWE und Veolia gegenüber zum direkten Nachteilsausgleich.

\section{Die „Raub- und Beutegemeinschaft"}

\section{a) Entscheidungsblockade statt Letztentscheidungsrecht des Landes}

Der Verfassungsgerichtshof ging unzutreffend von der typischen Idealsituation aus, in der das verfassungsmäßige Gesetz der Legislative das Exekutivhandeln determiniert. Faktisch wurde aber das Konstrukt des „Holdingmodells“ zur Teilprivatisierung der BWB von Experten im „rechtsfreien Raum“ entwickelt. Seine gesetzliche und vertragliche Gestaltung wurde im Prozess der Teilprivatisierung parallel zwischen Senat und Investoren ausgehandelt. Dadurch entstand - bei Berücksichtigung der Interessen von relevanten Beteiligten im politischen Prozess (Senatsmitglieder, Koalition, „Experten“: Consultants und Anwaltskanzleien), der antizipierten Interessen von relevanten Stakeholdern (Beschäftigte und Gewerkschaft, Verbände) - schließlich ein durchsetzbares Privatisierungsprojekt, das mit dem Verfassungsgerichtsurteil seine endgültige Form gewann. Das Gericht war deshalb in gewisser Weise „blind“ für die Verflechtungen zwischen Teilprivatisierungsgesetz und den Verträgen, die die formalen Vorgaben des Gesetzes informell aufweichten und unterliefen.

Während der Verfassungsgerichtshof im Teilprivatisierungsgesetz die demokratische Legitimation der BWB-Organe und das Letztentscheidungsrecht formal gewahrt sah, führte die komplizierte, vertraglich austarierte Balance der Einflussnahme von Land und Privatinvestoren bei den Kompetenzen und der Besetzung der Unternehmensorgane ${ }^{39}$ faktisch zu einem permanenten Einigungszwang zwischen den beteiligten Seiten. Weder das Land noch die Konzerne RWE und Veolia können mit den ihnen zur Verfügung stehenden Kompetenzen die strategische Ausrichtung der BWB allein bestimmen. Zwar ist den privaten Anteilseignern im Rahmen des Konsortialvertrags die unternehmerische Führung eingeräumt. Dem Land Berlin steht aber mit den Vetooptionen in den Aufsichtsgremien von Holding und Anstalt eine starke Blockadeposition zu, die es gegen die privaten Anteilseigner in Stellung bringen kann. Mit einem „Letztentscheidungsrecht“ Berlins in zentralen Fragen der Anstalt, die das Verfassungsgericht fordert, hat das aber nichts zu tun.

39 Vgl. Hecker (2001), S. 275 ff., sowie ausführlich Hüesker (2011), S. 155 ff. 
Im Ergebnis führte der strukturelle Einigungszwang dazu, dass Land und private Anteilseigner regelmäßig zur Verständigung jenseits der Konzerngremien veranlasst sind. Das gilt auch für Veränderungen in der Struktur des Holdingmodells. Sie werden sich bei der strategischen Ausrichtung der BWB auf den „kleinsten gemeinsamen Nenner“ zur Wahrung ihrer Interessen einigen. Die Vorkehrungen des Teilprivatisierungsgesetzes zur Sicherung demokratischer Legitimationsanforderungen rücken angesichts dieser informellen Strukturdeterminanten völlig in den Hintergrund. Das belegt auch die Praxis der Unternehmenssteuerung bis in die Gegenwart: Der vom Verfassungsgericht so hoch gewichtete „Weisungsausschuss“ des Holdingvorstands ist bislang nicht ein einziges Mal zusammengetreten.

\section{b) Neuverteilung der Belastungen}

Nach Vollzug der Teilprivatisierung erschöpfte sich der Gestaltungswillen der 1999 wiedergewählten schwarz-roten Koalition unverzüglich. Die handelnden Akteure sahen sich nicht zur „Korrektur“ der verfassungsgerichtlich beanstandeten Tarifkalkulationsvorschriften veranlasst, nach dem Bankenskandal 2001 wurden sie abgewählt. Die privaten Anteilseigner konnten angesichts der Nachteilsausgleichspflicht Berlins aus $§ 23$ Abs. 7 Konsortialvertrag gelassen bleiben. Erst mit Auslaufen der künstlichen Stabilisierung des Wasserpreises Ende 2003 entstand für das Haushaltskrisenland die Notwendigkeit, die für nichtig erklärten Vorschriften des Teilprivatisierungsgesetzes durch rechtssichere Vorschriften zu ersetzen, wollte es die aus den Teilprivatisierungsverträgen resultierenden Gewinnansprüche nicht dauerhaft allein und zu Lasten des Etats schultern. Denn die Nichtigerklärung der Kalkulationsvorschriften ging auf Kosten des Landes, das die Gewinnerwartungen der Privaten zu erfüllen hatte - und sei es zulasten eigener erwarteter Einnahmen.

2002 begann die neue rot-rote Regierung Verhandlungen mit RWE und Veolia, die schließlich zur Novellierung des Teilprivatisierungsgesetzes ${ }^{40}$ und zur Verabschiedung der 5. Änderungsvereinbarung zum Konsortialvertrag führten. ${ }^{41}$ Dem Senat wurde nun die Befugnis zum Erlass einer Rechtsverordnung eingeräumt, um eine adäquate Verzinsungsquote des betriebsnotwendigen Kapitals jährlich neu festsetzen zu können. Das schuf Spielraum für Preissteigerungen. Die Abschreibungsregelungen für das BWB-Anlagevermögen wurden auf Wiederbeschaffungszeitwerte umgestellt. All diese Änderungen unterlagen dem faktischen Konsenszwang mit RWE und Veolia und dienten der Kompensation der verfassungsgerichtlich beanstandeten Kalkulationsnormen ${ }^{42}$ in Erfüllung von $\S 23$ Abs. 7 des Konsortialvertrags. ${ }^{43}$ Der Korridor der 1999 eingegangenen Verpflichtungen gegenüber den privaten Anteilseignern stand dabei nicht grundsätzlich zur Disposition. Dies hätte den Konsens des Landes Berlin mit RWE und Veolia erfordert.

40 Gesetz zur Änderung des Teilprivatisierungsgesetzes vom 11.12.2003, GVB1. S. 591.

41 Anders als 1999 wurden die Inhalte und Konsequenzen der anstehenden Entscheidungen in Plenum und Ausschüssen breit diskutiert und konnten auch von allen Abgeordneten nachvollzogen werden. Das zeigt auch die Plenardebatte; vgl. Plenarprotokoll 15/42 der 42. Sitzung des Abgeordnetenhauses vom 11.12.2003.

42 Der Verfassungsgerichtshof Berlin hat die Verfassungskonformität der novellierten Kalkulationsvorschriften inzwischen bestätigt; vgl. BerlVerfGH 29/07 und 39/09, Beschlüsse vom 14.7.2010.

43 Vgl. hierzu auch Hüesker (2011), S. 173 ff. 
Die Teilprivatisierung von 1999 hatte eine „Raub- und Beutegemeinschaft“ der Anteilseigner zu Lasten der Wasserkunden hervorgebracht. Nichts verdeutlichte das stärker als das Eingeständnis der Abgeordneten in der Parlamentsdebatte zur Verabschiedung von Gesetznovelle und Änderungsvereinbarung, bei der Neujustierung der Gesetzesnormen im bestehenden Vertragskorridor an ihre Grenze geraten zu sein. Bemerkenswert ist, dass die Redner ausnahmslos aller Fraktionen die Teilprivatisierung 1999 als schweren politischen Fehler bezeichneten. In ihrer 2. Koalitionsvereinbarung ${ }^{44}$ von 2006 erklärten SPD und LINKE die „Rekommunalisierung der BWB“ sogar zum politischen Ziel ihrer Regierungsarbeit.

\section{c) Aufräumen bei den Beteiligungen, Effektivitätsdruck bei den BWB}

Folge der Teilprivatisierung waren seit 2004 stetig steigende Wasserpreise, denen dreistellige Millionen-Gewinnabführungen an die Anteilseigner gegenüberstanden. Im Rahmen der „kondominialen Leitungsstruktur ${ }^{645}$ der BWB wurden aber auch Entscheidungen getroffen, die nicht durch die Teilprivatisierungsverträge von 1999 determiniert waren. Anders, als 1999 intendiert, wurde das Wettbewerbsgeschäft der BWB weitgehend einvernehmlich abgewickelt. Die Zeit der Experimente war vorbei, nüchterne Rechnungen traten an die Stelle des Größenwahns der 1990er Jahre. Die Anteilseigner konzentrierten sich auf das Kerngeschäft der BWB. Das Interesse des Landes zielte auf Preisstabilität (trotz der faktischen Bindung der Entgeltkalkulation an die Renditeerwartungen) und gute Arbeitsverhältnisse für die Beschäftigten. Durch Einführung eines neuen Tarifmodells (Grundpreis, Arbeitspreis) und die Einführung eines Anschluss- und Benutzungszwangs wurden 2006 die Preiskalkulation der realen Kostenstruktur angenähert und die Solidarfunktion der Ver- und Entsorgungsinfrastruktur der BWB gestärkt. ${ }^{46}$ In diesen Verhandlungen mussten auch die privaten Anteilseigner Zugeständnisse machen, die mit Abstrichen bei der Rendite verbunden waren.

\section{Fazit}

Das alles kann aber nicht darüber hinwegtäuschen, dass die Zahl ungelöster Konflikte zwischen den Anteilseignern zunimmt, deren einvernehmliche Klärung im Rahmen der bestehenden Strukturen schwer erscheint. Es läuft ein Schiedsverfahren zur Gewinnverteilung zwischen Land und Privaten aufgrund der 5. Änderungsvereinbarung. Streit herrscht auch zum Umgang mit tarifrelevanten Faktoren, bei deren Ansatz die Verträge Spielräume eröffnen. Das Land verfolgt inzwischen seine Interessen gegenüber den Privaten stringenter und konfliktbereiter. Bei all diesen Auseinandersetzungen geht es letztlich um die Frage, ob Preissenkungen oder die Gewinnmaximierung in der Geschäftspolitik der BWB Vorrang haben sollen.

Der jüngste Konflikt ist erstmals von existenzieller Natur für das Teilprivatisierungsprojekt: Auf Anregung des (scheidenden) Aufsichtsratsvorsitzenden hat das Bundeskartellamt ein Verfahren

44 Koalitionsvereinbarung 2006-2011 zwischen der SPD und der Linkspartei.PDS vom 20.11.2006.

45 So Hecker (2001), S. 275.

46 Vgl. Berliner Betriebegesetz in der Fassung der Bekanntmachung vom 14.7.2006; GVB1. S. 827. 
wegen Preisüberhebung gegen die BWB eingeleitet. ${ }^{47}$ Das wurde damit begründet, dass die BWB - trotz vergleichbarer Kostenstrukturen - deutlich höhere Tarife verlangt als die Vergleichsunternehmen in München und Hamburg. Der Grund dafür ist tatsächlich der hohe Anteil kalkulatorischer Kosten in der Kalkulation zur Sicherung der Gewinne der Anteilseigner. Eine kartellamtliche Preissenkungsverfügung würde als Maßnahme aufgrund von Bundesrecht nicht den Nachteilsausgleichsmechanismus des $\S 23$ Abs. 7 Konsortialvertrag auslösen und damit die Geschäftsgrundlage des 1999er Teilprivatisierungsmodells ernsthaft in Frage stellen.

Es ist offen, wie all diese Konflikte aufgelöst werden, zumal Berlin nach der Abgeordnetenhauswahl vom 18. September 2011 vor einem rot-schwarzen Revival steht. Angesichts der politischen Debatten in der Stadt um die negativen Folgen der Teilprivatisierung hat RWE bereits 2010 Verhandlungsbereitschaft zur Rückübertragung der BWB-Anteile erkennen lassen. Veolia ist allerdings bislang nur zu einer „Modernisierung“ der vertraglichen Kooperationsbasis bereit ${ }^{48}$ und hat Interesse an einer Übernahme der RWE-Anteile angemeldet. Es gilt: Nur im Konsens der Beteiligten sind grundlegende Änderungen möglich. Das gilt auch für eine geänderte rechtliche Kooperationsgrundlage, die neue wettbewerbs- und vergaberechtliche Probleme aufwerfen würde und erneut einer parlamentarischen Legitimation bedürfte.

Das 1999 begründete BWB-Konstrukt ist kein Erfolgsmodell, sondern tatsächlich ein Abwicklungsfall. Die rechtliche Interessenverschränkung und faktische Steuerungsblockade zweier Seiten mit völlig unterschiedlichen Handlungslogiken ermöglicht keine konsistente Geschäftsstrategie, die die aktive Bewältigung permanent neu entstehender Probleme und Widersprüche erlaubt. Die BWB sind mit einem Tanker ohne Kommandobrücke vergleichbar. Das kann gutgehen, solange kein Eisberg die Fahrtroute kreuzt. Eine Rückabwicklung scheitert gegenwärtig - völlig unabhängig vom politischen Willen der jeweiligen Parlamentsmehrheit - daran, dass selbst die Beendigung des Projekts durch Berlin an der Zustimmung der privaten Partner hängt. Abschließend erlaube ich mir die Prognose, dass die existierende rechtliche Basis keine geeignete Grundlage für eine gedeihliche Kooperation über die gesamte vereinbarte Vertragslaufzeit darstellt. Schon vorher werden die inhärenten Reibungen und Interessengegensätze den starren rechtlichen Rahmen sprengen, weil sich in nüchterner Bilanz (inkl. Transaktionskosten, Image, Goodwill) für keine der beteiligten Seiten mehr lohnen wird, daran festzuhalten. Das gilt umso mehr, als der erfolgreiche Volksentscheid vom Februar 2011 zeigt, dass die Berlinerinnen und Berliner nicht länger bereit sind, für dieses Spiel den Preis zu zahlen. Ob daraus produktive Ansätze für Alternative zum Status quo erwachsen, bleibt abzuwarten. Aber schon jetzt diskutiert Berlin neue Ideen für die BWB.

47 Diese Möglichkeit eröffnete die Entscheidung des BGH, KVR 66/08, vom 6.2.2010, über die Anwendbarkeit der kartellrechtlichen Missbrauchsvorschriften auf Wasserpreise. Offen ist bislang, ob das auch für öffentlichrechtliche Unternehmen gilt, die anstelle von Gebühren Preise erheben. 
Die Teilprivatisierung der Berliner Wasserbetriebe: Erfolgsmodell oder Abwicklungsfall?

Abstract

Klaus Lederer; The partial privatization of Berlin's water supply company: Successful model or case for phaseout?

Public services; Public Supply and Disposal Enterprises; Public Corporate Governance; Partial Privatization; Municipal Water Infrastructures, Democratic Controlling

For a long time, the partial privatization of Berlin's water supply company (BWB) was regarged as a prime example for a successful public private partnership solution. In particular, it was emphasized that the privatization, which preserved the legal form of the company, has ideally combined the potentials of the corporate form under public law with the efficiency standards of the private sector. Twelve years after privatization, this article attempts to draw a provisional balance.

\section{Literaturverzeichnis}

Bezzenberger, Gerold und Detlev Schuster (1996), Die öffentliche Anstalt als abhängiges Konzernunternehmen, in: Zeitschrift für Gesellschaftsrecht (1996), S. 481-518.

Fett, Torsten (2000), Öffentlich-rechtliche Anstalten als abhängige Konzernunternehmen. Dargestellt unter besonderer Berücksichtigung des „Berliner Modells“ zur Konzernierung der Landesbank Berlin, Berlin.

Gesellschaft für öffentliche Wirtschaft (1993), Eigenbetrieb, Kapitalgesellschaft, Anstalt des öffentlichen Rechts Rechtsformänderung bei den Berliner Eigenbetrieben?, Beiträge zur öffentlichen Wirtschaft, Heft 12, Berlin.

Hecker, Jan (2001), Privatisierung unternehmenstragender Anstalten des öffentlichen Rechts - Anstaltsrechtliche, demokratiestaatliche und privatisierungstheoretische Überlegungen am Beispiel der Teilprivatisierung der Berliner Wasserbetriebe (BWB) -, in: Verwaltungsarchiv (2/2001), S. 261-291.

Hüesker, Frank (2011), Kommunale Daseinsvorsorge in der Wasserwirtschaft. Auswirkungen der Privatisierung am Beispiel der Wasserbetriebe Berlins, München.

Lederer, Klaus (2004), Strukturwandel bei kommunalen Wasserdienstleistungen. Eine Untersuchung aus verwaltungswissenschaftlicher Perspektive, Berlin.

Lederer, Klaus (2010), Die Teilprivatisierung der Berliner Wasserbetriebe - Chronologie eines Raubzugs. Dossier, in: Verkauftes Wasser, hrsg. von der Linksfraktion im Berliner Abgeordnetenhaus, Berlin, S. 2-10.

Mann, Thomas (1996), Die Entwicklung vom Eigenbetrieb zur rechtsfähigen Anstalt für Kommunalunternehmen, in: Zeitschrift für öffentliche und gemeinwirtschaftliche Unternehmen, Bd. 19, Heft 1, S. 53-63.

Ochmann, Daniela (2005), Rechtsformwahrende Privatisierung von öffentlich-rechtlichen Anstalten. Dargestellt am Holdingmodell zur Teilprivatisierung der Berliner Wasserbetriebe, Baden-Baden.

Rose, Matthew D. (1997), Berlin. Hauptstadt von Filz und Korruption, Hamburg.

Schmehl, Arndt (2001), Teilprivatisierung der Daseinsvorsorge, Demokratieprinzip und Gewinnerzielungsmaxime - BerlVerfGH, NVwZ 2000, 794, in: Juristische Schulung, Heft 3, S. 233-236.

Schoelkopf, Katrin (2010), RWE will über Anteilsverkauf verhandeln, in: Berliner Morgenpost vom 21. November 2010 , S. 9.

Schulte, Ewald B. (2002), 500 Millionen Euro Lehrgeld, in: Berliner Zeitung vom 1. Juli 2002, S. 33.

Schulte, Ewald B. (2004), Nur Wasser läuft gut, in: Berliner Zeitung vom 30. August 2004, S. 13.

Schwintowski, Hans-Peter (2001), Teilprivatisierung öffentlicher Anstalten - Grundsätze sachgerechter Gebührenberechnung, in: Zeitschrift für Neues Energierecht, 4. Jg., Heft 4, S. 254.

Senatsverwaltung für Finanzen (1999), Die Teilprivatisierung der BWB, Presseunterlagen zum Senatsbeschluss, 18. Juni 1999, Berlin.

Stuchtey, Bettina (2002), Wettbewerb auf dem Markt für leitungsgebundene Trinkwasserversorgung. Möglichkeiten und Grenzen, Baden-Baden.

Weinzen, Hans Willi (2000), Berlin und seine Finanzen. Eine Einführung in das Finanzwesen, 3., völlig neubearbeitete und stark erweiterte Auflage, Berlin.

Wolfers, Benedikt (2000), Privatisierung unter Wahrung der öffentlich-rechtlichen Rechtsform: Modellfall Berliner Wasserbetriebe, in: Neue Zeitschrift für Verwaltungsrecht, Heft 7, S. 765-767. 